\title{
La religiosité musulmane en actes. Une enquête auprès de citoyennes pieuses
}

Muslim religiosity in action. A survey of pious women citizens

La religiosidad musulmana en acción. Una encuesta sobre mujeres ciudadanas

piadosas

Anne-Sophie Lamine

\section{(2) OpenEdition}

Édition électronique

URL : https://journals.openedition.org/assr/38951

DOI : 10.4000/assr.38951

ISSN : $1777-5825$

Éditeur

Éditions de l'EHESS

Édition imprimée

Date de publication : 1 décembre 2018

Pagination : 99-107

ISSN : 0335-5985

\section{Référence électronique}

Anne-Sophie Lamine, «La religiosité musulmane en actes. Une enquête auprès de citoyennes

pieuses », Archives de sciences sociales des religions [En ligne], 184 | octobre-décembre 2018, mis en ligne le 01 janvier 2022, consulté le 12 janvier 2022. URL : http://journals.openedition.org/assr/38951 ; DOI : https://doi.org/10.4000/assr.38951 


\title{
La religiosité musulmane en actes
}

\section{Une enquête auprès de citoyennes pieuses}

\author{
À propos de: \\ Joulli Jeanette, Pious Practice and Secular Constraints. Women in \\ the Islamic Revival in Europe, Stanford, Stanford University Press, \\ 2015, 272 p.
}

L'ouvrage de Jeanette Jouili, professeure-assistante au département d'études religieuses de l'université de Pittsburgh, analyse finement les pratiques musulmanes pieuses de jeunes femmes allemandes et françaises, fréquentant des centres religieux dans la mouvance du renouveau islamique, à Cologne et en banlieue parisienne. Par ses apports empiriques et théoriques, il offre une contribution décisive à la sociologie et à l'anthropologie de l'islam européen. Il se base sur de longues enquêtes de terrain: entretiens et observations de cours de religion et d'autres pratiques quotidiennes, dans le cadre d'une recherche doctorale puis postdoctorale (entre 2002 et 2010). L'écriture particulièrement vivante et agréable se double d'une présentation sobre et efficace, incluant un index des notions et des noms. Le livre examine les difficultés et les efforts quotidiens de ces jeunes femmes "en quête d'une vie en accord avec leurs principes religieux dans le contexte des sociétés européennes sécularisées » (Jouili, 2015: 3). Le double objectif de l'auteure correspond aux deux parties principales: d'une part, observer comment se construisent les subjectivités islamiques pieuses (chapitres 2, 3 et 4) et d'autre part comment ces musulmanes font face aux contraintes séculières et en particulier à la stigmatisation des pratiques religieuses orthodoxes (chapitres 5 et 6).

Le premier chapitre, "Pratiquer l'islam dans des environnements inhospitaliers ", présente les terrains, la méthodologie et les appuis conceptuels. La qualité de l'exploration ethnographique s'allie en effet à une très solide culture théorique qui permet à l'auteure d'opérer des avancées innovantes. D'abord, elle maîtrise la filiation foucaldienne de l'analyse du travail sur soi : notamment Talal Asad (2003,2017) et Saba Mahmood (2009) qu'elle mobilise de façon originale pour analyser les subjectivités et la discipline de 
soi en contexte européen. Ensuite, elle revient finement sur l'éthique aristotélicienne et l'articule à l'anthropologie de l'éthique ordinaire développée par Michael Lambek (2010), afin d'examiner les négociations entre différents rôles sociaux et les rapports à l'espace public.

Précisons donc les concepts centraux utilisés et discutés dans cette étude. De Foucault, Jouili (2015: 15) retient l'éthique de soi, ces disciplines de soi éthiques qui "permettent à des individus d'effectuer [...] un certain nombre d'opérations sur leurs corps, leur âme, leurs pensées, leurs conduites, leurs modes d'être (Foucault, 2001: 1604), ainsi que la subjectivation, donc «la manière dont on doit se constituer soi-même comme sujet moral agissant en référence aux éléments prescriptifs qui constituent le code » (Foucault, 1984 : 33). À Mahmood analysant les femmes pieuses au Caire, elle emprunte l'idée d' "habiter la norme» (Mahmood, 2009: 32). En effet, les normes ne sont pas seulement construites ou déconstruites, renforcées ou subverties, elles peuvent aussi être "habitées", ce qui révèle la puissance d'agir des sujets. Mais, comme on le verra, Jouili se distingue de Mahmood, d'une part, en soulignant que ce travail sur soi n'est pas aussi linéaire et que les codes moraux ne sont pas aussi évidents (Jouili, 2015 : 15) et, d'autre part, en y ajoutant la notion d'empowerment, absente chez cette dernière. D'Asad, Jouili discute le concept de «tradition discursive», articulant le discours et la pratique: «Une tradition se compose essentiellement de discours visant à former des pratiquants quant à la forme appropriée et au but d'une pratique donnée qui, étant instituée, a une histoire » (Asad, 2017: 132). Asad précise sa définition: "Une tradition discursive islamique n'est rien d'autre qu'une tradition du discours musulman se reliant aux conceptions islamiques du passé et du futur, en référence à une pratique islamique particulière dans le présent ${ }^{1}$.» Les dimensions narrative et corporelle des pratiques sont donc intrinsèquement liées. Jouili souligne cependant que le contexte diasporique reconfigure la tradition discursive par la présence d'éléments de domination (discursive, idéologique et politique) qui introduisent des enjeux d'identités et de différence (Jouili, 2015: 13). En effet, du fait que "[leurs] pratiques sont discréditées, les musulmans religieux sont poussés à défendre leurs significations correctes" (ibid.: 12). Enfin, l'auteure mobilise le concept aristotélicien de phronesis, que l'on peut définir comme la sagesse pratique et le raisonnement moral, permettant de faire ce qui est juste dans une configuration et un contexte donné2.

La première partie de l'ouvrage, portant sur la formation de soi, s'ouvre par le deuxième chapitre, intitulé: "“Je veux m'instruire afin de renforcer ma foi”: se former dans les institutions islamiques ». Jouili décrit les expériences d'apprentissage religieux développées dans les structures liées au renouveau islamique. Elle souligne que, pour ses interlocutrices, «ce type de connaissances religieuses, à la fois formelles et acquises dans une démarche réflexive, est la

1. Ibid.; voir aussi Meziane, 2017.

2. On peut d'ailleurs souligner que cette approche praxéologique rejoint l'éthique pragmatiste deweyenne. 
condition sine qua non pour effectuer une transformation de soi complète, et donc pour adopter un véritable mode de vie islamique» (Jouili, 2015: 37). Cependant, la piété, la réflexivité et la subjectivité s'avèrent totalement intriquées au contexte séculier: "Les idéaux du sujet moderne - tels que le choix, la connaissance, la conscience de soi et la capacité de transformation de soi - ont créé un environnement discursif favorable à des traditions islamiques de travail sur soi [self-cultivation]» (ibid.). On comprend mieux dès lors l'accueil favorable d'éléments de la tradition soufie: "Cette insistance sur le travail sur soi - qui a souvent été portée par des savants musulmans, notamment de tradition soufie est devenue de plus en plus populaire dans les activités en plein développement des mouvements de renouveau islamique (aussi bien soufis qu'orthodoxes) à travers le monde" (ibid.: 37-38). Cette préoccupation est "omniprésente dans les discours des femmes et dans les enseignements des centres islamiques » (ibid.: 40). Elle correspond le plus souvent à l'expression arabe jihad al-nafs (lutte contre l'ego). Cette transformation de soi s'avère néanmoins fréquemment plus difficile que prévu. Une musulmane allemande, traductrice de profession et ayant suivi de nombreux cours de spiritualité, déplore: "Maintenant je stagne, malheureusement. Quand on travaille beaucoup, on n'a pas le temps d'étudier. Souvent, je préfère lire un roman plutôt que la Sira » (ibid.). Cette transformation de soi requiert, en effet, un effort continu, comme le souligne une travailleuse sociale qui est aussi enseignante pour une classe de femmes à Cologne, en insistant sur la relation entre foi et connaissance:

C'est comme dans n'importe quelle relation. Pour avoir une bonne relation, que ce soit comme épouse, comme amie, ou dans une relation parent-enfant, toutes les relations demandent un travail, un effort important. Pour la relation entre l'être humain et Dieu, c'est la même chose. C'est le travail sur cette relation entre l'humain et Dieu: par l'acquisition de la connaissance, tu te rapproches de Dieu. C'est l'interaction entre les aspects cognitifs et spirituels de la foi. D'un côté, il y a l'acquisition cognitive de la simple connaissance, les faits, les hadiths, les versets du Coran et la signification de leurs contenus. De l'autre côté, il y a un effet sur la relation avec Dieu. Plus j'ai de la connaissance, bien sûr, à condition que je sois convaincue de ces choses, plus je suis remplie de foi, plus je suis fière de ma foi et plus les racines sont profondes. Plus ma foi devient inébranlable. La croissance spirituelle est absolument liée à cette croissance de la connaissance, à laquelle on parvient par l'accès aux sources (ibid.: 41).

Elle associe le travail sur soi à un effort continu pour cultiver une relation avec le divin, le comparant à des relations entre proches. En lisant cela, on ne peut que faire un parallèle avec le beau livre d'Anne Gotman (2013) qui souligne aussi, à propos de croyants catholiques et juifs, que «les relations se cultivent avec Dieu comme avec tout humain » et qu'elles "requièrent un véritable investissement» et ajoute: "Parce que l'on croit, on y investit du temps et parce que l'on investit du temps, on croit » (ibid.: 88). Mais la connaissance ne suffit pas, comme le souligne encore une autre interviewée: "Si nous n'avons pas de spiritualité, que peut-on faire de cette connaissance dans notre vie quotidienne? Ma foi est nourrie par la connaissance et la spiritualité » 
(Jouili, 2015 : 42). L'anthropologue précise que cette connaissance va de pair avec le développement de capacités émotionnelles: «La connaissance ne doit pas seulement transmettre l'information, mais doit aussi aider la croyante à s'immerger dans une ambiance de présence divine. En retour, cette immersion doit faire naître des états émotionnels spécifiques, tels que les sentiments d'amour et de crainte [respectueuse] envers Dieu, l'humilité et la confiance en Dieu (tawakkul).» (ibid.: 43) Dès lors, l'objectif de cette connaissance (ilm) est de "cultiver des dispositions intérieures sensibles et incorporées [visceral and affective], qui se sédimentent, dans le long terme, en habitus islamique » (ibid.), par lesquelles la foi se construit et se renforce, et qui est donc liée aux sentiments de confiance en Dieu.

Le troisième chapitre s'intitule " "Ton Nafs [ego] te tire vers le bas, ton esprit vers le haut": les efforts vers la vertu». Il décrit les difficultés et les obstacles rencontrés dans ces efforts ainsi que la non-linéarité dans les efforts de réalisation du soi vertueux. Il montre comment la pédagogie élaborée dans les centres de formation fait une large place au raisonnement, à la réflexion et au questionnement sur les pratiques et les conduites. On y découvre que la prière est tout sauf facile, mais aussi que l'exigence de discipline va de pair avec une vision du divin dans laquelle l'amour prévaut et où les idées de peur et de punition sont à l'inverse totalement rejetées. Le port du voile, seconde forme essentielle de travail sur soi, est encouragé, tout en insistant sur l'importance de comprendre et de respecter celles qui ne le portent pas. La pudeur n'est cependant pas considérée comme l'apanage des femmes, mais au contraire comme l'affaire des deux genres. Dans ces efforts prévaut l'idée de faire son chemin et d'aimer Dieu et non celle du haram (illicite). Finalement, "le soi vertueux n'est pas un état, mais un idéal » et le travail sur soi est un «processus instable, fragile et constamment sujet à turbulence» (ibid.: 92).

Le quatrième chapitre, "Mon étiquette, ce n'est pas féministe, je suis simplement musulmane”: dépasser l'opposition entre émancipation et soumission, analyse de la dimension genrée du travail sur soi ", répond à cette question: comment ces femmes, qui ont grandi et se sont formées dans un contexte libéral, valorisant les droits individuels, parviennent-elles à dépasser cette opposition? Certaines recherches européennes montrent que l'acquisition du savoir est en soi une forme d'empowerment dans la mesure où il permet à ces femmes de critiquer les visions et pratiques patriarcales. Ces travaux considèrent donc implicitement que l'émancipation est "en cours» et que la contestation des normes genrées est à venir (ibid.: 94). Ce faisant, ils minimisent le désir d'éthique de soi vertueuse précédemment décrit. Dès lors, le recours à l'approche de Mahmood, déjà évoquée, permet de sortir de la dualité entre norme acceptée et norme contestée, entre autonomie et résistance à la subordination, avec sa proposition d'une troisième voie, celle d' « habiter la norme ». Cependant, Jouili, en travaillant dans le contexte européen, souligne une limite à la perspective mahmoodienne: "En se focalisant sur le désir de vertu et de piété, [cette approche] éclipse complètement le désir d'empowerment », très présent sur son terrain (ibid.: 95), notamment dans le désir de carrière et de prendre sa place 
dans la société. Il faut donc prendre en compte ces deux désirs: l'empowerment et la vertu. L'auteure observe en effet que l'effort pour articuler une vision de la dignité et de l'empowerment est impacté par le langage des droits de l'homme et de l'autonomie, tout en cherchant à s'en distancier. Comment articuler l'éthique de soi à une éthique de l'obligation, de la responsabilité et du care (ibid.: 96) ? Le thème du statut de la femme apparaît constamment dans les discours, avec une double stratégie discursive, basée sur la temporalité alternative de l'islam et sur la comparaison avec la femme de la chrétienté occidentale. On constate, sur ce terrain aussi, que le renvoi aux origines de la révélation religieuse, loin d'être l'apanage des seuls croyants fondamentalistes, peut être mobilisé de manières diversement réformistes. Le premier argument affirme « une temporalité islamique dans laquelle le stade ultime du progrès s'incarnerait dans la période médinoise, du vivant du Prophète. C'est à ce moment-là que la libération des femmes est comprise comme pleinement achevée, avec la mise en œuvre des droits des femmes tels qu'ils sont définis dans le Coran » (ibid.: 96), notamment par une certaine autonomie financière. Le second argument se base sur une comparaison avec l'histoire de la chrétienté, permettant d'appuyer une forme de retournement du stigmate. Emma, une Franco-marocaine, fait allusion à la loi du 13 juillet 1965 permettant aux femmes d'ouvrir un compte bancaire ou d'exercer une activité professionnelle sans l'aval de son conjoint: «En France, ce n'est qu'en 1965 que les femmes ont acquis le droit de disposer de leurs biens sans l'autorisation de leur mari... Nous, on a obtenu ce droit depuis quatorze siècles, depuis le début de l'islam»(ibid.: 97). Ce faisant, l'argument permet aussi de comprendre la tension vis-à-vis du religieux, comme le souligne la même interlocutrice: "Tu ne peux pas transposer [les critiques sur le traitement des femmes dans la chrétienté] à l'Islam, car l'Islam n'a pas la même histoire [...]. Si les femmes ont été opprimées pendant des siècles au nom de l'Église, je peux comprendre les débats passionnés qui ont cours maintenant» (ibid.: 98). Revendiquant une place plus juste pour les femmes dans leur groupe religieux, elles gardent cependant une distance par rapport à certaines formes de féminismes (y compris musulman), comme le souligne la Germano-turque Sevim: «Il y a des féministes qui sont un peu radicales. Dans notre centre nous sommes des femmes qui veulent réaliser quelque chose aussi. Nous sommes musulmanes et nous ne tombons pas en dehors du cadre du Coran ou de l'islam. Nous disons, OK, je suis peut-être à la limite du cadre, mais je suis encore dans le cadre. Et nous nous efforçons d'avancer dans la société avec cette conscience islamique» (ibid.: 103).

Si «l'Occident», «la chrétienté » et «l'Europe» sont des référents réactifs omniprésents, la réflexion n'en est pas moins travaillée par les visions éthiques de la tradition discursive islamique. Le passage des idéaux d'égalité, marqués par la vision libérale, à l'idéal musulman d'équité passe par l'acquisition de connaissances islamiques permettant à la fois de répondre à l'image négative de la femme musulmane et de construire un nouveau soi. Dans cet apprentissage, en se soumettant à la "sagesse divine », les femmes font face à des injonctions différenciées et inégalitaires, qu'elles questionnent fortement, «se situant 
dans un entrelacs de doute, de certitude, de réflexion et de confiance " (ibid.: 106). Jouili voit dans ce processus le changement du "rapport de soi à soi» (ibid.: 107), découlant d' "un changement de régime de discipline de soi», vers un régime "informé par les traditions islamiques, telles qu'elles ont été retravaillées dans les cercles de renouveau islamiques en Europe et qui ont aussi été clairement façonnées par le régime libéral de liberté que ces femmes veulent quitter en se tournant vers l'islam » (ibid.: 108). Dans ce processus complexe, elles s'appuient sur leurs dispositions émotionnelles, en particulier en insistant sur la confiance en Dieu: "Quand je vois ou j'entends des choses que je ne comprends pas, je les accepte, car j'estime que ma raison humaine est limitée. Dieu, qui a créé les êtres humains, comprend comment ils fonctionnent, il sait pourquoi » (ibid.: 110). Cela n'empêche nullement la solidarité sans consensus. Une des femmes a relaté "de nombreuses anecdotes sur ses luttes contre de "vieux hommes patriarcaux" lors de ses batailles pour favoriser la participation des femmes ou concernant leur place dans sa mosquée locale» (ibid.: 115). Un autre appui est l'application flexible et contextuelle des enseignements islamiques, en se focalisant sur les aspects considérés comme essentiels.

La seconde partie de l'ouvrage s'ouvre avec le cinquième chapitre, "Une femme au foyer diplômée ?": les femmes diplômées dans la famille et au travail ». Il aborde les dilemmes auxquels font face ces jeunes femmes pieuses, entre carrière et éducation des enfants. "Cherchez le savoir, même jusqu'en Chine ", ce hadith est souvent mobilisé pour souligner combien l'importance accordée à la connaissance et aux études est congruent avec la spiritualité musulmane. Comme le souligne une interviewée: "Si vous m’invitez à un séminaire sur l'écologie, cela m'intéresse, car pour moi c'est l'islam, même si vous ne l'appelez pas islam. Je veux dire, protéger la planète, c'est musulman, parler de la santé, c'est musulman. Tout est interconnecté » (ibid.: 124). Là encore, certaines femmes soulignent positivement leur différence par rapport à la chrétienté: "Quand il est dit dans le Coran "lis au nom de ton Seigneur", cela ne concerne pas seulement la connaissance du Coran et des Hadiths, cela concerne toute la connaissance. Le monde chrétien a été dogmatique pendant toute une période, c'est pour cela qu'ils sont contre la croyance maintenant». Elle fait allusion à la condamnation de Copernic par l'Église et ajoute: «En Islam c'est différent, plus tu as de connaissance, plus tu crois dans toutes les sciences » (ibid.: 125). Cette valorisation des études dans les centres islamiques pousse d'ailleurs certaines d'entre elles à retourner à l'université. Le devoir de la mère vis-à-vis de l'éducation n'en est pas moins valorisé, en ligne avec le discours réformiste. Il est cependant reconfiguré, notamment en le dissociant des tâches ménagères (ibid.: 128). En outre, le haut niveau d'études des femmes doit contribuer à une mobilité ascendante et faire émerger une nouvelle génération de musulmans européens (ibid.: 130). Une large majorité de ces femmes aspire à une vie professionnelle: "Une femme au foyer diplômée, non merci ». Leur approche islamique souligne l'interdépendance des droits et des devoirs. Mener une carrière professionnelle réussie est donc aussi vu comme un devoir, une responsabilité vis-à-vis de leur communauté et, plus globalement, de la 
société, en plus d'une opportunité de contrer l'image négative des musulmans. Ces femmes articulent ainsi leurs différents rôles à des éthiques de la sollicitude (famille, communauté, personnes fragiles, société). Ce sens du devoir, qui va de pair avec un fort désir personnel de vie professionnelle, est source de dilemmes moraux, comme le montre une très vive discussion et désapprobation faisant suite à la position conservatrice d'une enseignante, défendant la nécessité de rester au foyer lorsque les enfants sont jeunes. Il implique aussi un vif souhait de trouver un conjoint qui partage les tâches ménagères et facilite leur choix de carrière, nonobstant la critique des «nombreux hommes musulmans traditionnels » (ibid.: 150). L'articulation entre carrière et maternité est donc l'objet de négociations et de compromis. Là encore, on voit que le fait d'appartenir à une tradition particulière ne signifie pas l'absence de dilemmes, d'ambivalences et de conflits, mais requiert une constante délibération éthique, afin de trouver personnellement et pratiquement la juste manière d'agir.

Le sixième chapitre, "Musulmane visible: négocier sa présence dans les espaces publics ", montre que les manières d'agir vis-à-vis de cette tension diffèrent d'une femme à l'autre. Cependant, elles « relèvent toutes de la même compréhension de la responsabilité individuelle pour un bien commun plus large » (ibid.: 181-182). La vision musulmane du bien commun (maslaha) portée par ces femmes est très similaire à la catégorie morale grecque de phronesis, notamment par l'attention accordée au contexte dans le raisonnement éthique, qui se déploie dans une relation triangulaire entre ego, alter et Dieu ${ }^{3}$. Jouili s'appuie aussi sur l'anthropologie de «l'éthique ordinaire» développée par Lambek (2010), que nous pouvons rapprocher de l'idée de "religion ordinaire », bien que cet auteur ne travaille pas sur le religieux. Ce dernier recommande, pour comprendre "l'éthique ordinaire», "d'observer les pratiques en train de se faire, en incluant la manière donc les acteurs les décrivent », plutôt que «de tenter de discerner certaines vertus spécifiques ${ }^{4}$. La nécessité de cultiver la confiance en Dieu (tawakkul) et la patience ( $s a b r)$ est souvent soulignée, notamment lorsque ces femmes évoquent les difficultés dans leur cheminement religieux, en particulier les discriminations qu'elles subissent. Ce n'est cependant aucunement une résignation, mais un espace de puissance d'agir, incluant dévotion, raisonnement, travail sur soi et engagement dans la vie concrète (Jouili, 2015: 185), familiale, sociale, professionnelle, civique. «Les modes de raisonnement éthique mis en œuvre par ces femmes pieuses intègrent des éléments de raisonnement relevant à la fois du mode rationnel, de la contemplation, de l'observation, et qui se combinent à des émotions profondes (crainte, doute, compassion, amour, ambition et espoir). Des positions militantes ou des situations conflictuelles peuvent se produire, conjointement avec l'appel à l'aide divine et avec des inspirations provenant de prières istikhara [prière par laquelle la personne demande à Dieu d'être guidée face à un choix difficile] ou de rêves" (ibid.: 186).

3. Elle s'appuie sur Salvatore, 2007: 166.

4. Lambek, 2010: 20. Cette perspective rejoint aussi l'approche pragmatiste (deweyenne) de la valuation, se focalisant sur «ce à quoi les gens tiennent» et non sur des valeurs cristallisées. 
Le chapitre final est intitulé «Des citoyennes pieuses ». Une religiosité plus intensive laisse-t-elle encore de la place à cette dimension citoyenne? On peut penser au cas des mères musulmanes françaises privées de sorties scolaires ou de goûter de Noël et qui dans leur protestation se réfèrent aux droits de l'homme. Mais qu'en est-il du rapport au bien commun pour des personnes dont la piété est une composante plus centrale de leur mode d'existence? Un des éléments frappants et récurrents de l'enquête de Jouili est que ces femmes s'efforcent constamment de tenir une double exigence de piété et d'attention au monde commun. Cela est manifeste d'une manière générale dans leur conception du bien commun, mais aussi, en particulier, dans leur souci de donner une bonne image de l'islam. Cela apparaît surtout - ce qui peut paraître contre-intuitif à des observateurs extérieurs - dans leur préoccupation constante de ne pas choquer ou mettre mal à l'aise des personnes non religieuses ou ayant une autre forme de religiosité (notamment ne portant pas de signe religieux): «Elles espèrent, face au regard social constamment suspicieux, communiquer leur différence sous un éclairage plus positif, et traduire leur incompréhensible étrangeté en une différence "tolérable" ( (ibid.: 188).

Jouili montre à la fois comment les discours de ces Européennes musulmanes s'inscrivent dans un espace de «débat permanent et porteur de contestation interne» (ibid. : 189) et combien leurs manières d'habiter leurs normes religieuses sont marquées par ce contexte sécularisé. Elle insiste sur l'intrication de ces "divers modes de puissance d'agir, de réflexivité et de subjectivité » (ibid.: 190) qui facilitent l'attention à l'altérité non musulmane, le souci d'un bien commun bien plus large que celui de leur groupe et donc des "vertus civiques en contexte de pluralité » (ibid.). Elle souligne combien «leurs opinions, leurs discours, leurs subjectivités sont assurément modelés par divers modes de raisonnement, principes et concepts qui proviennent des diverses traditions discursives [religieuses et non religieuses] ainsi que de nombreuses expériences de situations concrètes» (ibid.: 191). Elle en conclut que ces femmes, dans leur effort permanent de tenir ensemble piété musulmane et attention au bien commun, peuvent être qualifiées de "citoyennes pieuses".

C’est bien la religiosité « en acte» et ses expressions, qui sont dans cet ouvrage finement observées et analysées. Les contradictions, les dilemmes et les questionnements, si souvent négligés dans les études sociologiques ou anthropologiques, sont subtilement décrits. L'approche d'auteur-e-s incontournables sur ce champ islamique, comme Mahmood, est judicieusement mobilisée et dépassée, alors que celle d'auteur-e-s ne travaillant pas sur le champ religieux, comme Lambek, est tout aussi pertinemment déployée. Cet ouvrage fera assurément date dans le champ de la sociologie et de l'anthropologie de l'islam européen.

Anne-Sophie LAmine

Université de Strasbourg, SAGE anne-sophie.lamine@unistra.fr 


\section{Bibliographie}

Asad Talal, 2003, Formations of the Secular: Christianity, Islam, Modernity, Stanford University Press.

-, 2017 [1986], «L'idée d'une anthropologie de l'islam » [ "The Idea of an Anthropology of Islam»], Archives de sciences sociales des religions, 180, p. 117-137.

Foucault Michel, 1984, Histoire de la Sexualité 2, L’Usage des Plaisirs, Paris, Gallimard. -, 2001, Dits et écrits II, 1976-1988, Paris, Gallimard.

Gotman Anne, 2013, Ce que la religion fait aux gens: sociologie des croyances intimes, Paris, Éditions de la Maison des sciences de l'homme.

Joulli Jeanette, 2015, Pious Practice and Secular Constraints. Women in the Islamic Revival in Europe, Stanford, Stanford University Press.

Lambeк Michael, 2010, Ordinary Ethics: Anthropology, Language, and Action, New York, Fordham University Press.

Mahmood Saba, 2009 [2005], Politique de la piété. Le féminisme à l'épreuve du renouveau islamique [Politics of Piety. The Islamic Revival and the Feminist Subject] Paris, La Découverte.

Meziane Mohamed Amer, 2017, "Comparer les traditions discursives ", Socio-anthropologie, 36. [En ligne]. http://journals.openedition.org/socio-anthropologie/3105.

Salvatore Armando, 2007, The Public Sphere: Liberal Modernity, Catholicism, Islam, New York, Palgrave Macmillan. 